\title{
The Role of Autophagy in Respiratory Complications of COVID-19
}

\author{
Shima Fakher ${ }^{1}$, Payam Peymani ${ }^{2}$, Saeid Ghavami (iD ${ }^{3}$ and Pooneh Mokarram (id ${ }^{4,}$ \\ ${ }^{1}$ Department of Biochemistry, Shiraz University of Medical Sciences, Shiraz, Iran \\ ${ }^{2}$ Health Policy Research Center, Shiraz University of Medical Sciences, Shiraz, Iran \\ ${ }^{3}$ Department of Human Anatomy and Cell Science, Rady Faculty of Health Sciences, Max Rady College of Medicine, University of Manitoba, Winnipeg, Canada \\ ${ }^{4}$ Autophagy Research Center, Shiraz University of Medical Sciences, Shiraz, Iran \\ "Corresponding author: Department of Biochemistry, Autophagy Research Center, Shiraz University of Medical Sciences, Shiraz, Iran. Email: mokaram2@gmail.com \\ Received 2020 March 22; Accepted 2020 April 03.
}

\begin{abstract}
Background: The rapid spreading of corona virus disease 2019 (COVID-19) worldwide results in pneumonia and acute respiratory distress syndrome in many patients, which can be the major cause of death in cases with COVID-19. It has been reported that chloroquine (CQ) has improved COVID-19-induced pneumonia in clinical trials.

Objectives: Since CQ and its derivatives are proved to exhibit anti-autophagy properties based on previous studies, autophagy can be introduced as a possible mechanism of respiratory complications.

Methods: In the current study, we reviewed papers of Google Scholar database with no time limitation.

Results: It was revealed that autophagy has an important role in the manifestation of COVID-19 respiratory complications

Conclusions: Autophagy is triggered by SARS-CoV2 virus for its replication and autophagy inhibitory treatments might be considered promising therapeutics.
\end{abstract}

Keywords: COVID-19, Autophagy, Chloroquine, Pneumonia, Therapeutics

\section{Background}

A severe acute respiratory syndrome was reported in December 2019 in Wuhan, China. Patients admitted to the central hospital with fever, cough, and dizziness underwent bronchoalveolar lavage RNA sequencing test. It was revealed that a new RNA virus from Coronaviridae family, was the pathologic source of this disease, which was named corona virus disease 2019 (COVID-19) by WHO (1). Up to 30 March 2020, 693,282 cases globally had been reported out of which 33,106 cases ended to death (2).

The common features of the disease in 41 confirmed patients in a hospital in Wuhan included abnormal chest CT results and pneumonia. Acute respiratory distress syndrome (29\%), RNAemia (15\%), acute cardiac injury (12\%), and secondary infection (10\%) were reported as their complications. Admission to ICU occurred in 32\% and resulted in death in $15 \%$ of the patients. The patients in ICU had elevated levels of interleukin-2 (IL-2), IL-7, IL-10, granulocyte-colony stimulating factor (G-CSF), interferon$\gamma$ inducible protein-10 (IP-10), monocyte chemoattractant protein-1 (MCP-1), macrophage inflammatory protein 1- $\alpha$ (MIP1- $\alpha)$, and tumor necrosis factor- $\alpha$ (TNF- $\alpha)$ in plasma (3).

The mortality of COVID-19 ranges from $2 \%$ to $20 \%$ with the highest risk in immunocompromised and older patients. The luminal side of the respiratory tract receives and releases viruses from the same side which can easily spread (4).

It was reported in China that chloroquine phosphate, previously used for the treatment of malaria had been safe and efficient for the treatment of COVID-19-based pneumonia (5).

\section{Objectives}

Since there is no general treatment or vaccine for COVID-19, and chloroquine, which is a typical antiautophagy drug, is an effective drug, we suggest that the possible mechanism of COVID-19 complications may be autophagy.

\section{Methods}

In the current study, we reviewed papers of Google Scholar database with no time limitation and a recent World Health Organization report. 


\section{Results and Discussion}

Chloroquine

[7-chloro-4-(4-diethylamino-1methylbutylamino)quinoline], exhibits antiinflammatory and anti-malarial characteristics (6). Hydroxychloroquine is also widely being used for its anti-inflammatory effects against rheumatoid arthritis and lupus erythematosus (7). The anti-inflammatory property of chloroquine is via the downregulation of the cell-mediated immune response and as an inhibitor of autophagy by blocking autolysosomes degradation (6).

Autophagy is a process in the cytosol performed by trapping materials in a double-membrane vesicle named autophagosome and transporting them to the lysosome to remove unwanted substrates. After lysosomal hydrolysis of the macromolecules, they are exported to the cytosol. These autophagy processes are mediated by conserved autophagy-related proteins (ATGs) (8).

Hydroxychloroquine as an analog of chloroquine with fewer drug interactions might be a potential treatment for COVID-19. It has been suggested that the drugs can change the $\mathrm{pH}$ of the cell membrane surface and inhibit virus-cell membrane fusion. They can also inhibit viral replication, viral proteins glycosylation, new virus particle transport and release. For COVID-19, a loading dose of $400 \mathrm{mg}$ hydroxychloroquine orally, and a maintenance dose of 200 $\mathrm{mg}$ is recommended for 4 days (9).

In a recent study conducted on French patients with COVID-19, $600 \mathrm{mg} /$ day of hydroxychloroquine was orally administrated and the nasopharyngeal viral load was evaluated. Azithromycin was also administrated in some experimental groups. After six days, 20 treated cases showed a significant decrease in the viral carriage compared to the controls. Azithromycin administration, in combination with hydroxychloroquine, suggests a strong synergistic effect to eliminate virus infection (10).

Autophagy increases the replication level of some RNA viruses like picornaviruses, which are single-stranded RNA viruses. In Atg5 deleted pancreatic acinar cells in mice, a dramatic decrease in the disease is observed in a model of coxsackievirus-induced pancreatitis. Multiple picornaviruses use ATGs while producing new virions, to generate membranes as a replication platform instead of being degraded. Generated autophagosomes in coxsackievirus B3 and enterovirus D68 fuse the plasma membrane-like secretory autophagy by viral proteases, which results in the exocytosis of virion-containing vesicles. These pseudoenveloped virions contain phosphatidylserine, which improves the penetrating into neighboring cells such as macrophages. That is how ATGs help the replication and cell penetration of Picornaviruses (8). It has been reported that the RNA level of hepatitis C virus (HCV) as an autophagy-inducing virus was decreased in the serum of HCV infected patients after 8 weeks of QC administration (11-13).

It has been revealed that hydrophilic pore (viroporin) formation in viroporins can result in IL- $\beta$ production by transporting $\mathrm{K}^{+}, \mathrm{H}^{+}$, and $\mathrm{Ca}^{2+}$ as well as reactive oxygen species, autophagy, and endoplasmic reticulum stress. Coronavirus envelope small membrane protein (E) has shown a cation-selective behavior that leads to excessive IL18 and IL-1 $\beta$ levels in the blood, lungs and lymphoid tissues of COVID-19 patients (14).

It is reported that most severe acute respiratory syndrome coronavirus 2 (SARS-CoV2) proteins might be evolutionarily constrained, except for the spike (S) protein. Antibodies for $S$ domains cause Fc receptor-mediated uptake leading to an alternative infection of phagocytic antigen presenting cells (APC), called antibody-dependent enhancement of disease (ADE). T cell depletion is observed in severe COVID-19 disease and may be exacerbated by APC infection. Many efforts at the production of either SARS-CoV1 vaccines, MERS-CoV vaccines, or SARS-CoV2 vaccines have encountered elevated risk of ADE induction through antibody infection of phagocytic immune cells (15).

In coronaviruses, the formation of replicative infectious particles in double-membrane vesicles (DMV) as well as autophagy, depend on ER-derived membranes. To the best of our knowledge, it can be suggested that coronaviruses interact with autophagy pathway components to utilize them for virus replication, and inhibit the autophagic flux. Substances inhibiting the generation of DMVs are reported to attenuate coronaviruses replication in vitro (16).

Considering these findings, it can be concluded that anti-autophagy therapeutics might improve respiratory complications in clinical trials and decrease COVID-19associated mortality.

\section{Acknowledgments}

We would like to thank all my colleagues at Autophagy Research Center.

\section{Footnotes}

Authors' Contribution: All authors contributed to writing the manuscript and selecting the subject.

Conflict of Interests: The authors declare no conflict of interest.

Funding/Support: None declared by the authors. 


\section{References}

1. Wu F, Zhao S, Yu B, Chen YM, Wang W, Song ZG, et al. A new coronavirus associated with human respiratory disease in China. $\mathrm{Na}$ ture. 2020;579(7798):265-9. doi: 10.1038/s41586-020-2008-3. [PubMed: 32015508]. [PubMed Central: PMC7094943].

2. World-Health-Organization. Coronavirus disease 2019 (COVID-19): Situation report. 2020.

3. Huang C, Wang Y, Li X, Ren L, Zhao J, Hu Y, et al. Clinical features of patients infected with 2019 novel coronavirus in Wuhan, China. Lancet. 2020;395(10223):497-506. doi: 10.1016/S0140-6736(20)30183-5. [PubMed: 31986264].

4. Hickok J. Antimicrobial photodisinfection therapy: Essential technology for infection control.2020. Available from: https://ondinebio.com/wpcontent/uploads/2020/03/Antimicrobial-Photodisinfection-

Therapy-White-Paper_02.28.2020.pdf.

5. Gao J, Tian Z, Yang X. Breakthrough: Chloroquine phosphate has shown apparent efficacy in treatment of COVID-19 associated pneumonia in clinical studies. Biosci Trends. 2020;14(1):72-3. doi: 10.5582/bst.2020.01047. [PubMed: 32074550].

6. Endy TP, Keiser PB, Cibula D, Abbott M, Ware L, Thomas SJ, et al. Effect of antimalarial drugs on the immune response to intramuscular rabies vaccination using a postexposure prophylaxis regimen. J Infect Dis. 2020;221(6):927-33. doi:10.1093/infdis/jiz558. [PubMed: 31743394]

7. Shippey EA, Wagler VD, Collamer AN. Hydroxychloroquine: An old drug with new relevance. Cleve Clin J Med. 2018;85(6):459-67. doi: 10.3949/ccjm.85a.17034. [PubMed: 29883308].

8. Keller MD, Torres VJ, Cadwell K. Autophagy and microbial pathogenesis. Cell Death Differ. 2020;27(3):872-86. doi: 10.1038/s41418-019-0481-8. [PubMed: 31896796].

9. Yao X, Ye F, Zhang M, Cui C, Huang B, Niu P, et al. In vitro antiviral activity and projection of optimized dosing design of hydroxychloroquine for the treatment of severe acute respiratory syndrome coro- navirus 2 (SARS-CoV-2). Clin Infect Dis. 2020. doi: 10.1093/cid/ciaa237. [PubMed: 32150618]. [PubMed Central: PMC7108130].

10. Gautret P, Lagier JC, Parola P, Hoang VT, Meddeb L, Mailhe M, et al. Hydroxychloroquine and azithromycin as a treatment of COVID19: Results of an open-label non-randomized clinical trial. Int J Antimicrob Agents. 2020:105949. doi: 10.1016/j.ijantimicag.2020.105949. [PubMed: 32205204]. [PubMed Central: PMC7102549].

11. Peymani P, Yeganeh B, Sabour S, Geramizadeh B, Fattahi MR, Keyvani $\mathrm{H}$, et al. New use of an old drug: Chloroquine reduces viral and ALT levels in HCV non-responders (a randomized, triple-blind, placebocontrolled pilot trial). Can J Physiol Pharmacol. 2016;94(6):613-9. doi: 10.1139/cjpp-2015-0507. [PubMed: 26998724].

12. Yeganeh B, Rezaei Moghadam A, Alizadeh J, Wiechec E, Alavian SM, Hashemi M, et al. Hepatitis B and C virus-induced hepatitis: Apoptosis, autophagy, and unfolded protein response. World J Gastroenterol. 2015;21(47):13225-39. doi: 10.3748/wjg.v21.i47.13225. [PubMed: 26715805]. [PubMed Central: PMC4679754].

13. Peymani P, Ghavami S, Yeganeh B, Tabrizi R, Sabour S, Geramizadeh B, et al. Effect of chloroquine on some clinical and biochemical parameters in non-response chronic hepatitis $C$ virus infection patients: Pilot clinical trial. Acta Biomed. 2016;87(1):46-53. [PubMed: 27163895].

14. Farag NS, Breitinger U, Breitinger HG, El Azizi MA. Viroporins and inflammasomes: A key to understand virus-induced inflammation. Int J Biochem Cell Biol. 2020;122:105738. doi: 10.1016/j.biocel.2020.105738. [PubMed: 32156572]. [PubMed Central: PMC7102644].

15. Ricke D, Malone RW. Medical countermeasures analysis of 2019-nCoV and vaccine risks for antibody-dependent enhancement (ADE). SSRN Electronic J. 2020. doi: 10.2139/ssrn.3546070.

16. Gassen NC, Niemeyer D, Muth D, Corman VM, Martinelli S, Gassen A, et al. SKP2 attenuates autophagy through Beclin1-ubiquitination and its inhibition reduces MERS-coronavirus infection. Nat Commun. 2019;10(1):5770. doi: 10.1038/s41467-019-13659-4. [PubMed: 31852899]. [PubMed Central: PMC6920372]. 